\title{
A Strong Unique Continuation Property for the Heat Operator with Hardy Type Potential
}

\author{
Agnid Banerjee ${ }^{1} \cdot$ Nicola Garofalo ${ }^{2} \cdot$ Ramesh Manna ${ }^{3}$
}

Received: 7 April 2020 / Published online: 3 August 2020

(c) The Author(s) 2020

\section{Abstract}

In this note we prove the strong unique continuation property at the origin for the solutions of the parabolic differential inequality

$$
\left|\Delta u-u_{t}\right| \leq \frac{M}{|x|^{2}}|u|,
$$

with the critical inverse square potential. Our main result sharpens a previous one of Vessella concerned with the subcritical case.

Keywords Strong unique continuation · Inverse square potential $\cdot$ Carleman estimates

Mathematics Subject Classification 35A02 $\cdot 35 \mathrm{~B} 60 \cdot 35 \mathrm{~K} 05$

\begin{abstract}
Agnid Banerjeeis supported in part by SERB Matrix Grant MTR/2018/000267 and by Department of Atomic Energy, Government of India, under Project No. 12-R \& D-TFR-5.01-0520. Nicola Garofalo is supported in part by the Progetto SID (Investimento Strategico di Dipartimento): "Non-local operators in geometry and in free boundary problems, and their connection with the applied sciences", University of Padova, 2017, and by the Progetto SID: "Non-local Sobolev and isoperimetric inequalities", University of Padova, 2019. Ramesh Manna is supported by C.V. Raman PDF, R(IA)CVR-PDF/2020/224.
\end{abstract}

$凶$ Nicola Garofalo

nicola.garofalo@unipd.it

Agnid Banerjee

agnid@ tifrbng.res.in

Ramesh Manna

rameshmanna@iisc.ac.in

1 Centre For Applicable Mathematics, Tata Institute of Fundamental Research, Bangalore 560065, India

2 Dipartimento di Ingegneria Civile, Edile e Ambientale (DICEA), Università di Padova, 35131 Padua, Italy

3 Department of Mathematics, Indian Institute of Science, Bangalore 560 012, India 


\section{Introduction}

The unique continuation property (ucp) for second order elliptic and parabolic equations represents one of the most fundamental aspects of pde's with a long history and several important ramifications. In this paper we prove the strong unique continuation property (sucp) for solutions to the parabolic differential inequality

$$
\left|\Delta u-u_{t}\right| \leq \frac{M}{|x|^{2}}|u|
$$

where $M>0$ is arbitrary. In [9] (see also [2]) Vessella proved a general sucp result for sub-critical parabolic equations of the type

$$
\left|\operatorname{div}(A(x, t) \nabla u)-u_{t}\right| \leq \frac{M}{|x|^{2-\delta}}|u|, \quad \delta>0
$$

under Lipschitz regularity assumptions on the principal part $A(x, t)$. This provided a parabolic counterpart to the previous work of Hörmander in [5]. Comparing (1.1) with (1.2), it is obvious that our result sharpens Vessella's theorem, when the latter is specialised to the heat equation.

As it is well-known, the inverse-square potential $V(x)=\frac{M}{|x|^{2}}$ represents a critical scaling threshold in quantum mechanics [1], and it is equally known that its singularity is the limiting case for the sucp for the differential inequality $|\Delta u| \leq \frac{M}{|x|^{m}}|u|$, see the counterexample in [3]. Such potential fails to be in $L_{l o c}^{n / 2}$, and in general does not have small $L^{n / 2, \infty}$ seminorm, thus in the context of the Laplacian the sucp cannot be treated by the celebrated result of Jerison and Kenig in [4] or the subsequent improvement by Stein in the appendix to the same paper. We recall that, in the time-independent case of the Laplacian, the sucp for the unrestricted inverse square potential was proved by Pan in [7]. One should also see Regbaoui [8] for further generalisations to variable coefficient equations and Lin et al. [6] for quantitative results.

The main new ingredient in this note is the following improved Carleman estimate for the heat operator $\Delta-\partial_{t}$ in a space-time cylinder that is tailor-made for the differential inequality (1.1). Such result replaces the corresponding sub-critical estimate in [9, Theorem 13] (see also [2, Theorem 2]). Similarly to the time-independent case in [7,8], our proof of Theorem 1.1 also exploits the spectral gap on $\mathbb{S}^{n-1}$. In addition, it relies in an essential way on a delicate a priori estimate which we prove in Lemma 2.2 below, and which we feel is of independent interest. We emphasise for the unfamiliar reader that, although related sub-critical estimates appear in the works [2], [9], the weight in our Carleman estimate (1.3) is different from that in such works, and a new result was required.

Theorem 1.1 Let $R<1$ and let $u \in C_{0}^{\infty}\left(\left(B_{R} \backslash\{0\}\right) \times(0, T)\right)$. There exist universal constants $\alpha(n)>>1$, and $\varepsilon(n) \in(0,1)$, such that for all $\alpha>\alpha(n)$ of the form $\alpha=k+\frac{n+1}{2}$, with $k \in \mathbb{N}$, and every $0<\varepsilon<\varepsilon(n)$, one has 


$$
\begin{aligned}
& \alpha \int_{B_{R} \times(0, T)}|x|^{-2 \alpha-4} e^{2 \alpha|x|^{\varepsilon}} u^{2} \mathrm{~d} x \mathrm{~d} t+\alpha^{3} \int_{B_{R} \times(0, T)}|x|^{-2 \alpha-4+\varepsilon} e^{2 \alpha|x|^{\varepsilon}} u^{2} \\
& \leq C \int_{B_{R} \times(0, T)}|x|^{-2 \alpha} e^{2 \alpha|x|^{\varepsilon}}\left(\Delta u-\partial_{t} u\right)^{2} \mathrm{~d} x \mathrm{~d} t
\end{aligned}
$$

where $C=C(\varepsilon, n)>0$.

With Theorem 1.1 in hands, we establish the following strong unique continuation result. In the sequel, parabolic vanishing to infinite order means that as $r \rightarrow 0$ one has for all $k>0$,

$$
\int_{B_{r} \times(0, T)} u^{2}=O\left(r^{k}\right) .
$$

Theorem 1.2 Suppose that for some $M, R, T>0$ the function $u \in H_{l o c}^{2,1}$ be a solution in $B_{R} \times(0, T)$ to the differential inequality (1.1). If u parabolically vanishes to infinite order, then $u \equiv 0$ in $B_{R} \times(0, T)$.

For the reader's comprehension we remark that the first integral in the left-hand side of (1.3) represents the critical term which, in the proof of Theorem 1.2, allows to absorb the term with the inverse square potential in the differential inequality (1.1). The second integral, instead, can be thought of as a sub-critical term. As the proof of Theorem 1.2 will show the presence of the coefficient $\alpha^{3}$ in front of such term plays a crucial role in the proof of Theorem 1.2, similarly to what happens in [9].

The plan of the paper is as follows. In Sect. 2 we prove Theorem 1.1. The second part of the section is devoted to proving the crucial Lemma 2.2 which is needed for the completion of the proof of Theorem 1.1. In Sect. 3 we establish Theorem 1.2. One word of caution for the reader. It is generally accepted among experts that, once a proper Carleman estimate is available, the ucp, or the sucp follow from a standard application of the former. While this is generally true, in the time-dependent setting of the present note deducing Theorem 1.2 from Theorem 1.1 requires a delicate adaptation of the analogous treatment of the subcritical case in [9]. For this reason, we have not followed the tradition of skipping details, but we have carefully presented them in the proof of Theorem 1.2.

\section{Proof of Theorem 1.1}

We begin by introducing the relevant notation. Given $r>0$ we denote by $B_{r}\left(x_{0}\right)$ the Euclidean ball centred at $x_{0} \in \mathbb{R}^{n}$ with radius $r$. When $x_{0}=0$, we will use the simpler notation $B_{r}$. A generic point in space time $\mathbb{R}^{n} \times(0, \infty)$ will be denoted by $(x, t)$. For notational convenience, $\nabla f$ and $\operatorname{div} f$ will, respectively, refer to the quantities $\nabla_{x} f$ and $\operatorname{div}_{x} f$ of a given function $f$. The partial derivative in $t$ will be denoted by $\partial_{t} f$ and also by $f_{t}$. We indicate with $C_{0}^{\infty}(\Omega)$ the set of compactly supported smooth functions in the region $\Omega$ in space-time. By $H_{l o c}^{2,1}(\Omega)$ we refer to the parabolic Sobolev class of functions $f \in L_{l o c}^{2}(\Omega)$ for which the weak derivatives $\nabla f, \nabla^{2} f$ and $\partial_{t} f$ belong to $L_{l o c}^{2}(\Omega)$. For a point $x \in \mathbb{R}^{n} \backslash\{0\}$, we will routinely adopt the notation 
$r=r(x)=|x|$ and $\omega=\frac{x}{r} \in \mathbb{S}^{n-1}$, so that $x=r \omega$. The radial derivative of a function $v$ is $v_{r}=<\nabla v, \frac{x}{|x|}>$.

The following simple observations will be repeatedly used in what follows. Let $\gamma \in \mathbb{R}$, then in $\mathbb{R}^{n} \backslash\{0\}$ we have

$$
\operatorname{div}\left(r^{-\gamma} x\right)=(n-\gamma) r^{-\gamma}
$$

In particular, (2.1) gives

Lemma 2.1 Let $f \in C_{0}^{\infty}\left(\mathbb{R}^{n} \backslash\{0\}\right), g \in C^{\infty}\left(\mathbb{R}^{n} \backslash\{0\}\right)$, then

$$
\int_{\mathbb{R}^{n}} f_{r} g \mathrm{~d} x=-\int_{\mathbb{R}^{n}} f g_{r} \mathrm{~d} x-(n-1) \int_{\mathbb{R}^{n}} r^{-1} f g \mathrm{~d} x .
$$

Proof It suffices to observe that (2.1) gives

$$
\operatorname{div}\left(f g r^{-1} x\right)=f g_{r}+g f_{r}+(n-1) r^{-1} f g .
$$

Integrating this identity we reach the desired conclusion.

Proof of Theorem 1.1 In all subsequent integrals, for given $R \in(0,1), T>0$, the domain of integration will be the parabolic cylinder $B_{R} \times(0, T)$ (or, for that matter, the whole of $\mathbb{R}^{n} \times \mathbb{R}$, in view of the support property of the integrands), but this will not be explicitly indicated. Nor, we will explicitly write the measure $\mathrm{d} x \mathrm{~d} t$ in any of the integrals involved. Let $u \in C_{0}^{\infty}\left(\left(B_{R} \backslash\{0\}\right) \times(0, T)\right)$. We set $v=r^{-\beta} e^{\alpha r^{\varepsilon}} u$, where $\beta$ is to be carefully chosen subsequently. Clearly, $u=r^{\beta} e^{-\alpha r^{\varepsilon}} v$. A standard calculation shows

$$
\Delta\left(r^{\beta} e^{-\alpha r^{\varepsilon}}\right)=\left(\alpha^{2} \varepsilon^{2} r^{\beta+2 \varepsilon-2}+\beta(\beta+n-2) r^{\beta-2}-\alpha \varepsilon((2 \beta+\varepsilon+n-2)) r^{\beta+\varepsilon-2}\right) e^{-\alpha r^{\varepsilon}} .
$$

We thus have

$$
\begin{aligned}
\Delta u & =r^{\beta} e^{-\alpha r^{\varepsilon}} \Delta v+\left(\alpha^{2} \varepsilon^{2} r^{\beta+2 \varepsilon-2}+\beta(\beta+n-2) r^{\beta-2}-\alpha \varepsilon((2 \beta+\varepsilon+n-2)) r^{\beta+\varepsilon-2}\right) e^{-\alpha r^{\varepsilon}} v \\
& +\left(2 \beta r^{\beta-2}-2 \varepsilon \alpha r^{\beta+\varepsilon-2}\right) e^{-\alpha r^{\varepsilon}}<x, \nabla v>.
\end{aligned}
$$

Since $\Delta v(x, t)=v_{r r}(r \omega, t)+\frac{n-1}{r} v_{r}(r \omega, t)+\frac{1}{r^{2}} \Delta_{\mathbb{S}^{n-1}} v(r \omega, t)$, where $\omega \in \mathbb{S}^{n-1}$ and $\Delta_{\mathbb{S}^{n-1}}$ denotes the Laplacian on $\mathbb{S}^{n-1}$, we obtain

$$
\begin{aligned}
& \Delta u-\partial_{t} u=r^{\beta} e^{-\alpha r^{\varepsilon}}\left[\left(\alpha^{2} \varepsilon^{2} r^{2 \varepsilon-2}+\beta(\beta+n-2) r^{-2}-\alpha \varepsilon(2 \beta+\varepsilon+n-2) r^{\varepsilon-2}\right) v\right. \\
& \left.\quad+\left((2 \beta+n-1) r^{-1}-2 \alpha \varepsilon r^{\varepsilon-1}\right) v_{r}+r^{-2} \Delta_{\mathbb{S}^{n-1}} v+v_{r r}-v_{t}\right]
\end{aligned}
$$


We now apply the numerical inequality $(a+b)^{2} \geq a^{2}+2 a b$, with

$$
a=r^{\beta-2} e^{-\alpha r^{\varepsilon}}\left(\beta(\beta+n-2) v+\Delta_{\mathbb{S}^{n-1}} v+(2 \beta+n-1) r v_{r}\right)
$$

and

$b=r^{\beta} e^{-\alpha r^{\varepsilon}}\left(\alpha^{2} \varepsilon^{2} r^{2 \varepsilon-2} v-\alpha \varepsilon(2 \beta+\varepsilon+n-2) r^{\varepsilon-2} v-2 \alpha \varepsilon r^{\varepsilon-1} v_{r}+v_{r r}-v_{t}\right)$,

obtaining

$$
\begin{aligned}
& \int r^{-2 \alpha} e^{2 \alpha r^{\varepsilon}}\left(\Delta u-\partial_{t} u\right)^{2} \geq \int r^{-2 \alpha+2 \beta-4}\left(\beta(\beta+n-2) v+\Delta_{\mathbb{S}^{n-1}} v\right)^{2} \\
& +(2 \beta+n-1)^{2} \int r^{-2 \alpha+2 \beta-2} v_{r}^{2}+2(2 \beta+n-1) \int r^{-2 \alpha+2 \beta-3} v_{r} \Delta_{\mathbb{S}^{n-1}} v \\
& +2 \beta(\beta+n-2)(2 \beta+n-1) \int r^{-2 \alpha+2 \beta-3} v v_{r} \\
& +2 \beta(\beta+n-2) \int r^{-2 \alpha+2 \beta-2} v v_{r r}+2 \int r^{-2 \alpha+2 \beta-2} v_{r r} \Delta_{\mathbb{S}^{n-1}} v \\
& +2(2 \beta+n-1) \int r^{-2 \alpha+2 \beta-1} v_{r} v_{r r}-2 \beta(\beta+n-2) \int r^{-2 \alpha+2 \beta-2} v v_{t} \\
& -2 \int r^{-2 \alpha+2 \beta-2} v_{t} \Delta_{\mathbb{S}^{n-1}} v-2(2 \beta+n-1) \int r^{-2 \alpha+2 \beta-1} v_{t} v_{r} \\
& -2 \alpha \varepsilon(2 \beta+n-1)(2 \beta+\varepsilon+n-2) \int r^{-2 \alpha+2 \beta+\varepsilon-3} v v_{r}+2 \alpha^{2} \varepsilon^{2}(2 \beta+n-1) \\
& \int r^{-2 \alpha+2 \beta+2 \varepsilon-3} v v_{r} \\
& -4 \alpha \varepsilon(2 \beta+n-1) \int r^{-2 \alpha+2 \beta+\varepsilon-2} v_{r}^{2}-4 \alpha \varepsilon \beta(\beta+n-2) \int r^{-2 \alpha+2 \beta+\varepsilon-3} v v_{r} \\
& -2 \alpha \varepsilon \beta(\beta+n-2)(2 \beta+\varepsilon+n-2) \\
& \int r^{-2 \alpha+2 \beta+\varepsilon-4} v^{2}+2 \alpha^{2} \varepsilon^{2} \beta(\beta+n-2) \int r^{-2 \alpha+2 \beta+2 \varepsilon-4} v^{2} \\
& -4 \alpha \varepsilon \int r^{-2 \alpha+2 \beta+\varepsilon-3} v_{r} \Delta_{\mathbb{S}^{n-1}} v-2 \alpha \varepsilon(2 \beta+\varepsilon+n-2) \int r^{-2 \alpha+2 \beta+\varepsilon-4} v \Delta_{\mathbb{S}^{n-1}} v \\
& +2 \alpha^{2} \varepsilon^{2} \int r^{-2 \alpha+2 \beta+2 \varepsilon-4} v \Delta_{\mathbb{S}^{n-1}} v \text {. }
\end{aligned}
$$

We now handle each integral in the right-hand side of (2.3) separately. Our first objective is to select $\beta$ in such a way that the integral $\int r^{-2 \alpha+2 \beta-3} v v_{r}$ vanishes. We note that such integral multiplies the cubic factor $2 \beta(\beta+n-2)(2 \beta+n-1)$ in the forth term in the right-hand side of (2.3). To accomplish this we observe that Lemma 2.1 gives 


$$
\begin{aligned}
& 2 \int r^{-2 \alpha+2 \beta-3} v v_{r}=\int r^{-2 \alpha+2 \beta-3}\left(v^{2}\right)_{r} \\
& =-(-2 \alpha+2 \beta-3) \int r^{-2 \alpha+2 \beta-4} v^{2}-(n-1) \int r^{-2 \alpha+2 \beta-4} v^{2}=0,
\end{aligned}
$$

provided that we choose

$$
2 \beta-2 \alpha-4=-n \Longleftrightarrow \beta=\alpha+2-\frac{n}{2}
$$

We now substitute the value of $\beta$ given by (2.5) in the remaining integrals in the right-hand side of (2.3) obtaining the following conclusions. First, we have

$$
\int r^{-2 \alpha+2 \beta-2} v v_{t}=\int r^{-n+2} v v_{t}=\int \partial_{t}\left(r^{-n+2} v^{2}\right)=0 .
$$

Next, using polar coordinates and Stokes' theorem on $\mathbb{S}^{n-1}$, we find

$$
\begin{aligned}
& 2 \int r^{-2 \alpha+2 \beta-2} v_{t} \Delta_{\mathbb{S}^{n-1}} v=2 \int r^{-n+2} v_{t} \Delta_{\mathbb{S}^{n-1}} v=2 \int_{0}^{T} \int_{0}^{\infty} r \int_{\mathbb{S}^{n-1}} v_{t} \Delta_{\mathbb{S}^{n-1}} v \mathrm{~d} \omega \mathrm{d} r \mathrm{~d} t \\
& =-2 \int_{0}^{T} \int_{0}^{\infty} r \int_{\mathbb{S}^{n-1}}<\nabla_{\mathbb{S}^{n-1}} v, \nabla_{\mathbb{S}^{n-1}} v_{t}>\mathrm{d} r \mathrm{~d} \omega \mathrm{d} t \\
& =-\int_{0}^{\infty} r \int_{0}^{T} \partial_{t}\left(\int_{\mathbb{S}^{n-1}}\left|\nabla_{\mathbb{S}^{n-1}} v\right|^{2} \mathrm{~d} \omega\right) \mathrm{d} t \mathrm{~d} r=0 .
\end{aligned}
$$

Similarly, we have

$$
\begin{aligned}
& 2 \int r^{-2 \alpha+2 \beta-3} v_{r} \Delta_{\mathbb{S}^{n-1}} v=2 \int r^{-n+1} v_{r} \Delta_{\mathbb{S}^{n-1}} v \\
& =2 \int_{0}^{T} \int_{0}^{\infty} \int_{\mathbb{S}^{n-1}} v_{r} \Delta_{\mathbb{S}^{n-1}} v \mathrm{~d} \omega \mathrm{d} r \mathrm{~d} t=-2 \int_{0}^{T} \int_{0}^{\infty} \int_{\mathbb{S}^{n-1}}\left\langle\nabla_{\mathbb{S}^{n-1}} v_{r}, \nabla_{\mathbb{S}^{n-1}} v\right\rangle \mathrm{d} \omega \mathrm{d} r \mathrm{~d} t \\
& =-\int_{0}^{T} \int_{0}^{\infty} \frac{\mathrm{d}}{\mathrm{d} r} \int_{\mathbb{S}^{n-1}}\left|\nabla_{\mathbb{S}^{n-1}} v\right|^{2} \mathrm{~d} \omega \mathrm{d} r \mathrm{~d} t=0
\end{aligned}
$$

Now, an integration by parts similar to (2.6) gives

$$
-4 \alpha \varepsilon \int r^{-2 \alpha+2 \beta+\varepsilon-3} v_{r} \Delta_{\mathbb{S}^{n-1}} v=-2 \alpha \varepsilon^{2} \int r^{-n+\varepsilon}\left|\nabla_{\mathbb{S}^{n-1}} v\right|^{2} .
$$

On the other hand, applying again the divergence theorem on $\mathbb{S}^{n-1}$, we find

$$
\begin{aligned}
& -2 \alpha \varepsilon(2 \beta+\varepsilon+n-2) \int r^{-2 \alpha+2 \beta+\varepsilon-4} v \Delta_{\mathbb{S}^{n-1}} v \\
& =2 \alpha \varepsilon(2 \alpha+\varepsilon+2) \int r^{-n+\varepsilon}\left|\nabla_{\mathbb{S}^{n-1}} v\right|^{2}
\end{aligned}
$$


and

$$
2 \alpha^{2} \varepsilon^{2} \int r^{-2 \alpha+2 \beta+2 \varepsilon-4} v \Delta_{\mathbb{S}^{n-1}} v=-2 \alpha^{2} \varepsilon^{2} \int r^{-n+2 \varepsilon}\left|\nabla_{\mathbb{S}^{n-1}} v\right|^{2} .
$$

Keeping in mind that on the domain of integration we have $0<r \leq R<1$, from (2.7), (2.8) and (2.9) we deduce that if $\varepsilon$ is sufficiently small, for instance, $0<\varepsilon \leq \frac{3}{20}$ would do, we can guarantee that

$$
\begin{aligned}
& -4 \varepsilon \alpha \int r^{-2 \alpha+2 \beta+\varepsilon-3} v_{r} \Delta_{\mathbb{S}^{n-1}} v-2 \alpha \varepsilon(2 \beta+\varepsilon+n-2) \int r^{-2 \alpha+2 \beta+\varepsilon-4} v \Delta_{\mathbb{S}^{n-1}} v \\
& +2 \alpha^{2} \varepsilon^{2} \int r^{-2 \alpha+2 \beta+2 \varepsilon-4} v \Delta_{\mathbb{S}^{n-1}} v \geq \frac{37}{10} \alpha^{2} \varepsilon \int r^{-n+\varepsilon}\left|\nabla_{\mathbb{S}^{n-1}} v\right|^{2}
\end{aligned}
$$

We also claim that

$$
\int r^{-2 \alpha+2 \beta-2} v v_{r r}=\int r^{-n+2} v v_{r r}=-\int r^{-n+2} v_{r}^{2} .
$$

To see (2.11) we apply Lemma 2.1 with $g=r^{-n+2} v$ and $f=v_{r}$, obtaining

$$
\int r^{-2 \alpha+2 \beta-2} v v_{r r}=\int r^{-n+2} v v_{r r}=-\int r^{-n+2} v_{r}^{2}+(n-2) \int r^{-n+1} v v_{r} .
$$

Since the last term vanishes in view of (2.4), we conclude that (2.11) holds. Next, again by Lemma 2.1 we have

$$
\int r^{-2 \alpha+2 \beta-1} v_{r} v_{r r}=\frac{1}{2} \int r^{-n+3}\left(v_{r}^{2}\right)_{r}=-\int r^{-n+2} v_{r}^{2} .
$$

Yet another application of Lemma 2.1 gives

$$
\begin{aligned}
& 2 \int r^{-2 \alpha+2 \beta-2} v_{r r} \Delta_{\mathbb{S}^{n-1}} v=2 \int r^{-n+2} v_{r r} \Delta_{\mathbb{S}^{n-1}} v \\
& =-2 \int r^{-n+2} v_{r} \Delta_{\mathbb{S}^{n-1}} v_{r}-2(n-1) \int r^{-n+1} v_{r} \Delta_{\mathbb{S}^{n-1}} v \\
& =2 \int_{0}^{T} \int_{0}^{\infty} r \int_{\mathbb{S}^{n-1}}\left|\nabla_{\mathbb{S}^{n-1}} v_{r}\right|^{2} \mathrm{~d} \omega \mathrm{d} r \mathrm{~d} t \geq 0 .
\end{aligned}
$$

Note that in the third equality above we have used that $\int r^{-n+1} \Delta_{\mathbb{S}^{n-1}} v v_{r}=0$, a fact which was earlier established in (2.6).

A further application of Lemma 2.1 gives

$$
2 \int r^{-2 \alpha+2 \beta+\varepsilon-3} v v_{r}=-\varepsilon \int r^{-n+\varepsilon} v^{2}
$$


Using this observation along with (2.5) we obtain

$$
\begin{aligned}
& -2 \alpha \varepsilon(2 \beta+n-1)(2 \beta+\varepsilon+n-2) \int r^{-2 \alpha+2 \beta+\varepsilon-3} v v_{r}+2 \alpha^{2} \varepsilon^{2}(2 \beta+n-1) \\
& \int r^{-2 \alpha+2 \beta+2 \varepsilon-3} v v_{r} \\
& -4 \alpha \varepsilon \beta(\beta+n-2) \int r^{-2 \alpha+2 \beta+\varepsilon-3} v v_{r} \\
& =\alpha \varepsilon^{2}[(2 \alpha+\varepsilon)(2 \alpha+\varepsilon+2)-\alpha \varepsilon(2 \alpha+3)+(2 \alpha+4-n)(2 \alpha+n)] \int r^{-n+\varepsilon} v^{2} .
\end{aligned}
$$

On the other hand, again by (2.5) we have

$$
\begin{aligned}
& -2 \alpha \varepsilon \beta(\beta+n-2)(2 \beta+\varepsilon+n-2) \\
& \int r^{-2 \alpha+2 \beta+\varepsilon-4} v^{2}+2 \alpha^{2} \varepsilon^{2} \beta(\beta+n-2) \int r^{-2 \alpha+2 \beta+2 \varepsilon-4} v^{2} \\
& =-\frac{\alpha \varepsilon}{2}(2 \alpha+4-n)(2 \alpha+n)\left(2 \alpha+2+\varepsilon\left(1-\frac{\alpha}{2}\right)\right) \int r^{-n+2 \varepsilon} v^{2} .
\end{aligned}
$$

Combining the latter two observations we conclude that there exists a universal constant $C>0$ such that, if $0<\varepsilon \leq \frac{3}{20}$ as above, and $\alpha>1$ is sufficiently large depending on the dimension $n$, then

$$
\begin{aligned}
& -2 \alpha \varepsilon(2 \beta+n-1)(2 \beta+\varepsilon+n-2) \int r^{-2 \alpha+2 \beta+\varepsilon-3} v v_{r}+2 \alpha^{2} \varepsilon^{2}(2 \beta+n-1) \\
& \int r^{-2 \alpha+2 \beta+2 \varepsilon-3} v v_{r} \\
& -4 \alpha \varepsilon \beta(\beta+n-2) \int r^{-2 \alpha+2 \beta+\varepsilon-3} v v_{r} \\
& -2 \alpha \varepsilon \beta(\beta+n-2)(2 \beta+\varepsilon+n-2) \int r^{-2 \alpha+2 \beta+\varepsilon-4} v^{2}+2 \alpha^{2} \varepsilon^{2} \beta(\beta+n-2) \\
& \int r^{-2 \alpha+2 \beta+2 \varepsilon-4} v^{2} \\
& \geq-C \alpha^{4} \varepsilon \int r^{-n+\varepsilon} v^{2} .
\end{aligned}
$$

We also note that by further restricting $\varepsilon$, say $0<\varepsilon \leq \frac{1}{20}$, we can ensure by (2.5) that for $\alpha>1$ we have

$$
4 \alpha \varepsilon(2 \beta+n-1) \int r^{-2 \alpha+2 \beta+\varepsilon-2} v_{r}^{2}=4 \alpha \varepsilon(2 \alpha+3) \int r^{-n+2+\varepsilon} v_{r}^{2} \leq \alpha^{2} \int r^{-n+2} v_{r}^{2}
$$


From (2.12), (2.15) and (2.5) once again, we infer the following estimate for $\alpha$ large enough (say, $\alpha \geq 10$ )

$$
\begin{aligned}
& (2 \beta+n-1)^{2} \int r^{-2 \alpha+2 \beta-2} v_{r}^{2}+2(2 \beta+n-1) \int r^{-2 \alpha+2 \beta-1} v_{r} v_{r r} \\
& -4 \alpha \varepsilon(2 \beta+n-1) \int r^{-2 \alpha+2 \beta+\varepsilon-2} v_{r}^{2} \\
& \geq\left[(2 \alpha+3)^{2}-2(2 \alpha+3)-\alpha^{2}\right] \int r^{-n+2} v_{r}^{2} \geq 2 \alpha^{2} \int r^{-n+2} v_{r}^{2} .
\end{aligned}
$$

The tenth integral in the right-hand side of (2.3) is simply handled as follows

$$
\begin{aligned}
& \left|2(2 \beta+n-1) \int r^{-2 \alpha+2 \beta-1} v_{t} v_{r}\right| \leq 4 \alpha\left(1+\frac{3}{2 \alpha}\right) \int r^{-n+3}\left|v_{t}\right|\left|v_{r}\right| \\
& \leq 5 \alpha\left(\frac{\alpha}{5} \int r^{-n+2} v_{r}^{2}+\frac{5}{\alpha} \int r^{-n+4} v_{t}^{2}\right) \leq \alpha^{2} \int r^{-n+2} v_{r}^{2}+25 \int r^{-n+4} v_{t}^{2} .
\end{aligned}
$$

Finally, we handle the first integral in the right-hand side of (2.3). We stress that such integral accounts for the critical term in the Carleman estimate (1.3). Recall that in the Fourier decomposition of $L^{2}\left(\mathbb{S}^{n-1}\right)$, if $Y_{k}(\omega)$ is a spherical harmonic of degree $k \in \mathbb{N} \cup\{0\}$ (that we assume normalised so that $\int_{\mathbb{S}^{n-1}}\left|Y_{k}(\omega)\right|^{2} \mathrm{~d} \omega=1$ ), then $\Delta_{\mathbb{S}^{n-1}} Y_{k}=-k(k+n-2) Y_{k}$. Therefore, if we write $v(x, t)=v(r \omega, t)$, and we indicate with $v_{k}(r, t)=\int_{\mathbb{S}^{n-1}} v(r \omega, t) Y_{k}(\omega) \mathrm{d} \omega$ its $k$-th Fourier coefficient in the Fourier decomposition $v(r \omega, t)=\sum_{k=0}^{\infty} v_{k}(r, t) Y_{k}(\omega)$, then we have

$$
\Delta_{\mathbb{S}^{n-1}} v(r \omega, t)=-\sum_{k=0}^{\infty} k(k+n-2) v_{k}(r, t) Y_{k}(\omega)
$$

Using this representation and Parseval's theorem, we obtain

$$
\begin{aligned}
& \int r^{-2 \alpha+2 \beta-4}\left(\beta(\beta+n-2) v+\Delta_{\mathbb{S}^{n-1}} v\right)^{2}=\int r^{-n}\left(\beta(\beta+n-2) v+\Delta_{\mathbb{S}^{n-1}} v\right)^{2} \\
& =\int_{0}^{T} \int_{0}^{\infty} r^{-1} \sum_{k=0}^{\infty}(\beta(\beta+n-2)-k(k+n-2))^{2} v_{k}(r, t)^{2} \mathrm{~d} r \mathrm{~d} t
\end{aligned}
$$

At this point, we assume that $\operatorname{dist}(\beta, \mathbb{N})=1 / 2$. Since for every $k \in \mathbb{N} \cup\{0\}$ we have

$$
(\beta(\beta+n-2)-k(k+n-2))^{2}=((\beta-k)(\beta+k+n-2))^{2} \geq \frac{1}{4}\left(\alpha+\frac{2 k+n}{2}\right)^{2},
$$

we thus infer 


$$
\begin{aligned}
& \int r^{-n}\left(\beta(\beta+n-2) v+\Delta_{\mathbb{S}^{n-1}} v\right)^{2} \\
& \geq \frac{\alpha^{2}}{4} \int_{0}^{T} \int_{0}^{\infty} r^{-1} \sum_{k=0}^{\infty} v_{k}(r, t)^{2} \mathrm{~d} r \mathrm{~d} t=\frac{\alpha^{2}}{4} \int r^{-n} v^{2}
\end{aligned}
$$

We now observe that, in view of (2.5), we have $\beta=k+\frac{1}{2}$ for some $k \in \mathbb{N}$ if and only if $\alpha=k-2+\frac{n+1}{2}$. This shows that for $\alpha$ large (depending on $n$ )

$$
\operatorname{dist}(\beta, \mathbb{N})=\frac{1}{2} \Longleftrightarrow \alpha=k+\frac{n+1}{2}, \text { for some } k \in \mathbb{N} \text {. }
$$

Combining (2.3)-(2.18), we reach the conclusion that there exists a universal $C>0$ such that for all $\alpha>1$ sufficiently large (depending on $n$ ) and as in (2.19), and for every $0<\varepsilon \leq \frac{1}{20}$, one has

$$
\begin{aligned}
& \int r^{-2 \alpha} e^{2 \alpha r^{\varepsilon}}\left(\Delta u-u_{t}\right)^{2} \geq \frac{\alpha^{2}}{4} \int r^{-n} v^{2}+2 \alpha^{2} \int r^{-n+2} v_{r}^{2}+\frac{37}{10} \alpha^{2} \varepsilon \int r^{-n+\varepsilon}\left|\nabla_{\mathbb{S}^{n-1}} v\right|^{2} \\
& -\alpha^{2} \int r^{-n+2} v_{r}^{2}-25 \int r^{-n+4} v_{t}^{2}-C \alpha^{4} \varepsilon \int r^{-n+\varepsilon} v^{2} \\
& =\frac{\alpha^{2}}{4} \int r^{-n} v^{2}+\alpha^{2} \int r^{-n+2} v_{r}^{2}+\frac{37}{10} \alpha^{2} \varepsilon \int r^{-n+\varepsilon}\left|\nabla_{\mathbb{S}^{n-1}} v\right|^{2} \\
& -25 \int r^{-n+4} v_{t}^{2}-C \alpha^{4} \varepsilon \int r^{-n+\varepsilon} v^{2} . \\
& \geq \frac{\alpha^{2}}{4} \int r^{-n} v^{2}-25 \int r^{-n+4} v_{t}^{2}-C \alpha^{4} \varepsilon \int r^{-n+\varepsilon} v^{2} .
\end{aligned}
$$

Recalling (2.5), and that $v=r^{-\beta} e^{\alpha r^{\varepsilon}} u$, we conclude that we have established the following bound

$$
\begin{aligned}
& \int r^{-2 \alpha} e^{2 \alpha r^{\varepsilon}}\left(\Delta u-u_{t}\right)^{2} \geq \frac{\alpha^{2}}{4} \int r^{-2 \alpha-4} e^{2 \alpha r^{\varepsilon}} u^{2} \\
& -25 \int r^{-2 \alpha} e^{2 \alpha r^{\varepsilon}} u_{t}^{2}-C \alpha^{4} \varepsilon \int r^{-2 \alpha-4+\varepsilon} e^{2 \alpha r^{\varepsilon}} u^{2}
\end{aligned}
$$

Keeping in mind that our final objective is proving (1.3), we mention at this point that the two negative terms in the right-hand side of (2.20) represent a series obstruction toward such goal. To overcome such difficulty we will establish the following delicate a priori bound. We stress that, differently from (2.20), the spectral gap assumption (2.19) is not needed.

Lemma 2.2 Let $R<1$ and let $u \in C_{0}^{\infty}\left(\left(B_{R} \backslash\{0\}\right) \times(0, T)\right)$. There exist constants $C_{0}=C_{0}(n)>0, \alpha(n)>>1$ and $0<\varepsilon(n)<<1$, such that for all $\alpha \geq \alpha(n)$ and every $0<\varepsilon<\varepsilon(n)$ one has 


$$
\frac{C_{0}}{\alpha} \int r^{-2 \alpha} e^{2 \alpha r^{\varepsilon}} u_{t}^{2}+C_{0} \alpha^{3} \varepsilon^{2} \int r^{-2 \alpha-4+\varepsilon} e^{2 \alpha r^{\varepsilon}} u^{2} \leq \int r^{-2 \alpha} e^{2 \alpha r^{\varepsilon}}\left(\Delta u-u_{t}\right)^{2} .
$$

The proof of Lemma 2.2 is postponed to the end of the section. With such result in hands we now proceed to complete the proof of Theorem 1.1. We fix $0<\varepsilon(n)<1$ and $\alpha(n)>>1$ such that (2.20) and (2.21) hold simultaneously for $0<\varepsilon<\varepsilon(n)$ and $\alpha>\alpha(n)$ and satisfying (2.19). We then choose and fix $\varepsilon \in(0, \varepsilon(n))$. Corresponding to such a choice of $\varepsilon$, we now select $C_{2}=C_{2}(n, \varepsilon)>1$ such that

$$
C_{2} C_{0} \varepsilon \geq 2 C \text { and } C_{2} C_{0}>25 .
$$

With such constant $C_{2}$ in hands, we multiply (2.21) by $C_{2} \alpha$ and add the resulting inequality to $(2.20)$, obtaining

$$
\begin{aligned}
& \frac{\alpha^{2}}{4} \int r^{-2 \alpha-4} e^{2 \alpha r^{\varepsilon}} u^{2}+\left(C_{2} C_{0} \varepsilon-C\right) \alpha^{4} \varepsilon \int r^{-2 \alpha-4+\varepsilon} e^{2 \alpha r^{\varepsilon}} u^{2} \\
& +\left(C_{2} C_{0}-25\right) \int r^{-2 \alpha} e^{2 \alpha r^{\varepsilon}} u_{t}^{2} \leq\left(C_{2} \alpha+1\right) \int r^{-2 \alpha} e^{2 \alpha r^{\varepsilon}}\left(\Delta u-u_{t}\right)^{2} .
\end{aligned}
$$

By our choice of $C_{2}$, and after dividing through by $\alpha$, the following inequality easily follows from (2.22)

$\alpha \int r^{-2 \alpha-4} e^{2 \alpha r^{\varepsilon}} u^{2}+\alpha^{3} \int r^{-2 \alpha-4+\varepsilon} e^{2 \alpha r^{\varepsilon}} u^{2} \leq \frac{2 C_{2}}{\min \{1 / 4, C \varepsilon\}} \int r^{-2 \alpha} e^{2 \alpha r^{\varepsilon}}\left(\Delta u-u_{t}\right)^{2}$.

Modulo Lemma 2.2, this completes the proof of the Carleman estimate (1.3).

We now turn to the

Proof of Lemma 2.2 The proof of the estimate (2.21) is somewhat delicate. Letting as before $v=r^{-\beta} e^{\alpha r^{\varepsilon}} u$, at first we write the expression of $\Delta u-u_{t}$ in (2.2) in the form

$$
\Delta u-u_{t}=a+b
$$

with

$$
a=r^{\beta} e^{-\alpha r^{\varepsilon}}\left(v_{r r}+B(r, \alpha, \beta) v+r^{-2} \Delta_{\mathbb{S}^{n-1}} v\right),
$$

and

$$
b=r^{\beta} e^{-\alpha r^{\varepsilon}}\left(A(r, \alpha, \beta) v_{r}-v_{t}\right),
$$

where

$$
\left\{\begin{array}{l}
B(r, \alpha, \beta)=\left(\alpha^{2} \varepsilon^{2} r^{2 \varepsilon-2}+\beta(\beta+n-2) r^{-2}-\alpha \varepsilon(2 \beta+\varepsilon+n-2) r^{\varepsilon-2}\right), \\
A(r, \alpha, \beta)=(2 \beta+n-1) r^{-1}-2 \alpha \varepsilon r^{\varepsilon-1}
\end{array}\right.
$$


In what follows, for the sake of brevity we simply write $A$ and $B$, instead of $A(r, \alpha, \beta)$ and $B(r, \alpha, \beta)$. Also, the Eq. (2.5) will be repeatedly used without further reference to it. For instance, we note that, using such equation, we have from (2.23) the following alternative expression of $A$ and $B$

$$
\left\{\begin{array}{l}
B=\left(\alpha^{2} \varepsilon^{2} r^{2 \varepsilon-2}+\alpha^{2}(1+o(1)) r^{-2}-2 \alpha^{2} \varepsilon(1+o(1)) r^{\varepsilon-2}\right) \\
A=2 \alpha(1+o(1)) r^{-1}-2 \alpha \varepsilon r^{\varepsilon-1}
\end{array}\right.
$$

where we have denoted with $o(1)$ quantities which do not depend of $r$ and such that $|o(1)| \rightarrow 0$ as $\alpha \rightarrow \infty$. The reader should note that among the ensuing computations that lead to (2.21), some are similar to those of (2.3)-(2.18), and therefore several details will be skipped.

With this being said, our strategy is to expand $\left(\Delta u-u_{t}\right)^{2}=a^{2}+b^{2}+2 a b$, and then estimate from below each of the corresponding integrals $\int r^{-2 \alpha} e^{2 \alpha r^{\varepsilon}} a^{2}$, $\int r^{-2 \alpha} e^{2 \alpha r^{\varepsilon}} b^{2}$, and $2 \int r^{-2 \alpha} e^{2 \alpha r^{\varepsilon}} a b$ in an appropriate way. We begin with

$$
\begin{aligned}
& 2 \int r^{-2 \alpha} e^{2 \alpha r^{\varepsilon}} a b=2 \int A r^{-2 \alpha+2 \beta} v_{r} v_{r r}+2 \int A B r^{-2 \alpha+2 \beta} v v_{r} \\
& +2 \int A r^{-2 \alpha+2 \beta-2} v_{r} \Delta_{\mathbb{S}^{n-1}} v-2 \int r^{-2 \alpha+2 \beta} v_{t}\left(v_{r r}+B v+r^{-2} \Delta_{\mathbb{S}^{n-1}} v\right),
\end{aligned}
$$

and estimate each term that appears in the right-hand side of (2.25) separately. By Lemma 2.1 we have

$$
\begin{aligned}
& 2 \int A r^{-2 \alpha+2 \beta} v_{r} v_{r r}=-2(2 \alpha+3) \iint r^{-n+2} v_{r}^{2} \\
& +2 \alpha \varepsilon(2-\varepsilon) \iint r^{-n+2+\varepsilon} v_{r}^{2} \geq-5 \alpha \int r^{-n+2} v_{r}^{2},
\end{aligned}
$$

provided $\alpha \geq 6$. Next, we find from (2.24)

$$
\begin{aligned}
& 2 \int A B r^{-2 \alpha+2 \beta} v v_{r}=4 \alpha^{3}(1+o(1)) \int r^{-n+1} v v_{r} \\
& +12 \alpha^{3} \varepsilon^{2}(1+o(1)) \int r^{-n+1+2 \varepsilon} v v_{r} \\
& -12 \alpha^{3} \varepsilon(1+o(1)) \int r^{-n+1+\varepsilon} v v_{r}-4 \alpha^{3} \varepsilon^{3} \int r^{-n+1+3 \varepsilon} v v_{r} .
\end{aligned}
$$

As in (2.4), we have

$$
\int r^{-n+1} v v_{r}=0
$$

A repeated application of Lemma 2.1, and the fact that $r \leq R<1$, give 


$$
\begin{aligned}
& 2 \int r^{-n+1+2 \varepsilon} v v_{r} \geq-2 \varepsilon \int r^{-n+\varepsilon} v^{2}, \\
& -2 \int r^{-n+1+\varepsilon} v v_{r}=\varepsilon \int r^{-n+\varepsilon} v^{2},
\end{aligned}
$$

and

$$
-2 \int r^{-n+1+3 \varepsilon} v v_{r}=3 \varepsilon \int r^{-n+3 \varepsilon} v^{2} \geq 0
$$

Using the latter four relations in (2.27), we conclude

$$
\begin{aligned}
& 2 \int A B r^{-2 \alpha+2 \beta} v v_{r} \geq\left[6 \alpha^{3} \varepsilon^{2}(1+o(1))-12 \alpha^{3} \varepsilon^{3}(1+o(1))\right] \int r^{-n+\varepsilon} v^{2} \\
& =6 \alpha^{3} \varepsilon^{2}(1-2 \varepsilon)(1+o(1)) \int r^{-n+\varepsilon} v^{2} .
\end{aligned}
$$

It is clear from this estimate that, if $0<\varepsilon<\frac{1}{240}$, then for $\alpha>>1$ sufficiently large we have

$$
2 \int A B r^{-2 \alpha+2 \beta} v v_{r} \geq \frac{59}{10} \alpha^{3} \varepsilon^{2} \int r^{-n+\varepsilon} v^{2} \text {. }
$$

Furthermore, we obtain from (2.24)

$$
\begin{aligned}
& 2 \int A r^{-2 \alpha+2 \beta-2} v_{r} \Delta_{\mathbb{S}^{n-1}} v=4 \alpha(1+o(1)) \int r^{-n+1} v_{r} \Delta_{\mathbb{S}^{n-1}} v \\
& -4 \alpha \varepsilon \int r^{-n+1+\varepsilon} v_{r} \Delta_{\mathbb{S}^{n-1}} v=-2 \alpha \varepsilon^{2} \int r^{-n+\varepsilon}\left|\nabla_{\mathbb{S}^{n-1}} v\right|^{2},
\end{aligned}
$$

where in the last equality we have used (2.6) and (2.7). We also have

$$
\begin{aligned}
& -2 \int r^{-2 \alpha+2 \beta} v_{t}\left(v_{r r}+B v+r^{-2} \Delta_{\mathbb{S}^{n-1}} v\right)=-2 \int r^{-n+4} v_{t} v_{r r} \\
& -2 \int B r^{-n+4} v v_{t}-2 \int r^{-n+2} v_{t} \Delta_{\mathbb{S}^{n-1}} v .
\end{aligned}
$$

The latter two integrals in the right-hand side vanish, similarly to the two computations following (2.5). Using instead Lemma 2.1, we find

$$
\begin{aligned}
& -2 \int r^{-n+4} v_{r r} v_{t}=6 \int r^{-n+3} v_{t} v_{r}+2 \int r^{-n+4} v_{r} v_{t r} \\
& =6 \int r^{-n+3} v_{t} v_{r}+\int \partial_{t}\left(r^{-n+4} v_{r}^{2}\right)=6 \int r^{-n+3} v_{t} v_{r}
\end{aligned}
$$

We deduce that

$$
-2 \int r^{-2 \alpha+2 \beta} v_{t}\left(v_{r r}+B v+r^{-2} \Delta_{\mathbb{S}^{n-1}} v\right)=6 \int r^{-n+3} v_{t} v_{r} .
$$


If we now combine (2.25)-(2.30) we conclude that

$$
\begin{aligned}
& 2 \int r^{-2 \alpha} e^{2 \alpha r^{2 \varepsilon}} a b \geq \frac{59}{10} \alpha^{3} \varepsilon^{2} \int r^{-n+\varepsilon} v^{2}+6 \int r^{-n+3} v_{t} v_{r} \\
& -2 \alpha \varepsilon^{2} \int r^{-n+\varepsilon}\left|\nabla_{\mathbb{S}^{n-1}} v\right|^{2}-5 \alpha \int r^{-n+2} v_{r}^{2}
\end{aligned}
$$

Our next objective is to eliminate the negative term $-2 \alpha \varepsilon^{2} \int r^{-n+\varepsilon}\left|\nabla_{\mathbb{S}^{n-1}} v\right|^{2}$ in the right hand side of (2.31). We stress that, although at a first glance it might seem that such integral could be absorbed by the above discarded positive term $\frac{37}{10} \alpha^{2} \varepsilon \int r^{-n+\varepsilon}\left|\nabla_{\mathbb{S}^{n-1}} v\right|^{2}$ in (2.20), a careful look at the analysis that led to (2.22) reveals that this would not work.

Having said this, to accomplish our objective we instead proceed with estimating from below $\int r^{-2 \alpha} e^{2 \alpha r^{\varepsilon}} a^{2}$ as follows

$$
\begin{aligned}
& \int r^{-2 \alpha} e^{2 \alpha r^{\varepsilon}} a^{2}=\int r^{-2 \alpha+2 \beta}\left(v_{r r}+B v+r^{-2} \Delta_{\mathbb{S}^{n-1}} v\right)^{2} \\
& =\int r^{-2 \alpha+2 \beta}\left(\left(v_{r r}+B v+r^{-2} \Delta_{\mathbb{S}^{n-1}} v+\alpha \varepsilon^{2} r^{\varepsilon-2} v\right)-\alpha \varepsilon^{2} r^{\varepsilon-2} v\right)^{2} \\
& \geq-2 \alpha \varepsilon^{2} \int r^{-n+2+\varepsilon} v\left(v_{r r}+\left(B+\alpha \varepsilon^{2} r^{\varepsilon-2}\right) v+r^{-2} \Delta_{\mathbb{S}^{n-1}} v\right)
\end{aligned}
$$

where in the last inequality above we have used $\left(c_{1}+c_{2}\right)^{2} \geq 2 c_{1} c_{2}$, with

$$
c_{1}=v_{r r}+\left(B+\alpha \varepsilon^{2} r^{\varepsilon-2}\right) v+r^{-2} \Delta_{\mathbb{S}^{n-1}} v, \quad c_{2}=-\alpha \varepsilon^{2} r^{\varepsilon-2} v
$$

We then estimate each term in the right-hand side of (2.32) as follows. First, the divergence theorem on $\mathbb{S}^{n-1}$ gives

$$
-2 \alpha \varepsilon^{2} \int r^{-n+\varepsilon} v \Delta_{\mathbb{S}^{n-1}} v=2 \alpha \varepsilon^{2} \int r^{-n+\varepsilon}\left|\nabla_{\mathbb{S}^{n-1}} v\right|^{2},
$$

which precisely eliminates the negative term $-2 \alpha \varepsilon^{2} \int r^{-n+\varepsilon}\left|\nabla_{\mathbb{S}^{n-1}} v\right|^{2}$ in (2.31), see (2.37) below. Secondly, a repeated application of Lemma 2.1 gives

$$
-2 \alpha \varepsilon^{2} \int r^{-n+2+\varepsilon} v v_{r r}=2 \alpha \varepsilon^{2} \int r^{-n+2+\varepsilon} v_{r}^{2}-\alpha \varepsilon^{3}(1+\varepsilon) \int r^{-n+\varepsilon} v^{2}
$$

Thirdly, using the expression of $B$ in (2.24) it is easily seen that for $\alpha>>1$ sufficiently large and $0<\varepsilon<\frac{1}{240}$ we have

$$
-2 \alpha \varepsilon^{2} \int r^{-n+2+\varepsilon}\left(B+\alpha \varepsilon^{2} r^{\varepsilon-2}\right) v^{2} \geq-3 \alpha^{3} \varepsilon^{2} \int r^{-n+\varepsilon} v^{2} .
$$


From (2.32)-(2.35) we infer that

$$
\int r^{-2 \alpha} e^{2 \alpha r^{\varepsilon}} a^{2} \geq 2 \alpha \varepsilon^{2} \int r^{-n+\varepsilon}\left|\nabla_{\mathbb{S}^{n-1}} v\right|^{2}-\alpha \varepsilon^{3}(1+\varepsilon) \int r^{-n+\varepsilon} v^{2}-3 \alpha^{3} \varepsilon^{2} \int r^{-n+\varepsilon} v^{2}
$$

Combining now (2.31) and (2.36), and keeping in mind that $b=r^{\beta} e^{-\alpha r^{\varepsilon}}\left(A v_{r}-v_{t}\right)$, we obtain

$$
\begin{aligned}
& \int r^{-2 \alpha} e^{2 \alpha r^{\varepsilon}}\left(\Delta u-u_{t}\right)^{2} \geq\left(\frac{29}{10} \alpha^{3} \varepsilon^{2}-\alpha \varepsilon^{3}(\varepsilon+1)\right) \int r^{-n+\varepsilon} v^{2}-5 \alpha \int r^{n+2} v_{r}^{2} \\
& +6 \int r^{-n+3} v_{t} v_{r}+\int r^{-n+2}\left(r A v_{r}-r v_{t}\right)^{2}
\end{aligned}
$$

Now, for $0<\varepsilon<\frac{1}{10}$ we have

$$
\frac{29}{10} \alpha^{3} \varepsilon^{2}-\alpha \varepsilon^{3}(\varepsilon+1) \geq 2 \alpha^{3} \varepsilon^{2}
$$

Using this in (2.37) above, we deduce the following estimate

$$
\begin{aligned}
& \int r^{-2 \alpha} e^{2 \alpha r^{\varepsilon}}\left(\Delta u-u_{t}\right)^{2} \geq 2 \alpha^{3} \varepsilon^{2} \int r^{-n+\varepsilon} v^{2}-5 \alpha \int r^{n+2} v_{r}^{2} \\
& +6 \int r^{-n+3} v_{t} v_{r}+\int r^{-n+2}\left(r A v_{r}-r v_{t}\right)^{2}
\end{aligned}
$$

Our next and final objective is to bound from below the right-hand side of (2.38) by an expression that includes $\frac{C_{0}}{\alpha} \int r^{-n+4} v_{t}^{2}$, as desired in (2.21). In the process, we also need to control the negative term $-5 \alpha \int r^{-n+2} v_{r}^{2}$. To achieve this we consider the last two terms in the right-hand side of (2.38) and, adapting a similar idea in [2,9], we estimate them in two different ways. First, we proceed as follows

$$
\begin{aligned}
\mathcal{R} & \stackrel{\text { def }}{=} 6 \int r^{-n+3} v_{t} v_{r}+\int r^{-n+2}\left(r A v_{r}-r v_{t}\right)^{2} \\
& =6 \int r^{-n+3} v_{t} v_{r}+\int r^{-n+2}\left((r A-3) v_{r}-r v_{t}+3 v_{r}\right)^{2} \\
& =\int r^{-n+2}(6 r A-9) v_{r}^{2}+\int r^{-n+2}\left((r A-3) v_{r}-r v_{t}\right)^{2} \\
& \geq \int r^{-n+2}(6 r A-9) v_{r}^{2} .
\end{aligned}
$$

Using (2.24), it is easy now to recognise that for $\alpha>>1$ large and $0<\varepsilon<\frac{1}{300}$, we have $r A \geq \frac{19}{10} \alpha$, and therefore $(6 r A-9) \geq \frac{114}{10} \alpha-9 \geq \frac{112}{10} \alpha$, by increasing further the value of $\alpha>>1$. In conclusion, we obtain

$$
\mathcal{R} \geq \frac{112}{10} \alpha \int r^{-n+2} v_{r}^{2} .
$$


Proceeding in a similar way, we recognise that

$$
\begin{aligned}
\mathcal{R}= & 6 \int r^{-n+3} v_{t} v_{r}+\int r^{-n+2}\left(r A v_{r}-r\left(1-\frac{3}{r A}\right) v_{t}-\frac{3}{A} v_{t}\right)^{2} \\
= & 6 \int r^{-n+3} v_{t} v_{r}+\int r^{-n+4}\left(A v_{r}-\left(1-\frac{3}{r A}\right) v_{t}\right)^{2} \\
& +9 \int r^{-n+2} \frac{1}{A^{2}} v_{t}^{2}-2 \int r^{-n+3}\left(A v_{r}-\left(1-\frac{3}{r A}\right) v_{t}\right) \frac{3}{A} v_{t} \\
\geq & \int r^{-n+4} \frac{1}{(r A)^{2}}(6 r A-9) v_{t}^{2} .
\end{aligned}
$$

At this point we note that (2.24) implies $r A \leq 2 \alpha(1+o(1)) \leq 3 \alpha$, for $\alpha>>1$ sufficiently large. Combining this with the previous estimate from below $6 r A-9 \geq$ $\frac{112}{10} \alpha$, we obtain $\frac{1}{(r A)^{2}}(6 r A-9) \geq \frac{1}{\alpha}$, for $\alpha>>1$ large and $0<\varepsilon<\frac{1}{300}$. This gives

$$
\mathcal{R} \geq \frac{1}{\alpha} \int r^{-n+4} v_{t}^{2}
$$

If we now split $\mathcal{R}=\frac{9}{10} \mathcal{R}+\frac{1}{10} \mathcal{R}$, and apply (2.39) to $\frac{9}{10} \mathcal{R}$, and (2.40) to $\frac{1}{10} \mathcal{R}$, we finally obtain

$$
\mathcal{R} \geq \frac{1008}{100} \alpha \int r^{-n+2} v_{r}^{2}+\frac{1}{10 \alpha} \int r^{-n+4} v_{t}^{2} .
$$

At this point we are almost done. Using the inequality (2.41) in (2.38), we obtain

$$
\begin{aligned}
& \int r^{-2 \alpha} e^{2 \alpha r^{\varepsilon}}\left(\Delta u-u_{t}\right)^{2} \geq 2 \alpha^{3} \varepsilon^{2} \int r^{-n+\varepsilon} v^{2} \\
& +\left(\frac{1008}{100}-5\right) \alpha \int r^{-n+2} v_{r}^{2}+\frac{1}{10 \alpha} \int r^{-n+4} v_{t}^{2} \\
& \geq 2 \alpha^{3} \varepsilon^{2} \int r^{-n+\varepsilon} v^{2}++\frac{1}{10 \alpha} \int r^{-n+4} v_{t}^{2}
\end{aligned}
$$

where we have taken advantage of the crucial gain in positivity of the coefficient of $\int r^{-n+2} v_{r}^{2}$. If we now keep in mind that $v=r^{-\beta} e^{\alpha r^{\varepsilon}} u$, we finally deduce that (2.21) holds.

\section{Proof of Theorem 1.2}

In this section we show how to obtain the sucp result in Theorem 1.2 from Theorem 1.1. With the new estimate (1.3) in hands, we can adapt to the critical differential inequality (1.1) some of the ideas that in [9, Theor. 15, pp. 658-664] were developed in the subcritical context of (1.2). As we have mentioned in the introduction, this 
entails a delicate modification of Vessella's proof. For this reason, and for the sake of the reader's comprehension, we will present a detailed account. We begin with the following simple Caccioppoli type inequality.

Lemma 3.1 Let $u$ be a solution to (1.1) in $B_{R} \times(-T, T)$ and let $0<a<1<b$. Then, there exists a constant $C_{1}>0$, depending on $n, a, b, T$ and $M$ in (1.1), such that for every $r<\min \{1, R\}$ the following holds

$$
\int_{\{r / 2<|x|<r\} \times(-T / 2, T / 2)}|\nabla u|^{2} \leq \frac{C_{1}}{r^{2}} \int_{\{r(1-a) / 2<|x|<b r\} \times(-T, T)} u^{2} .
$$

Proof From (1.1), we may assume that $u$ solves $\Delta u-u_{t}=V u$, where $|V(x, t)| \leq$ $\frac{M}{|x|^{2}}$. Let now $\phi \equiv 1$ in $\{r / 2<|x|<r\} \times(-T / 2, T / 2)$, and vanishing outside $\{r(1-a) / 2<|x|<b r\} \times(-T, T)$. Using $\phi^{2} u$ as a test function in the weak form of the equation we obtain

$$
\int|\nabla u|^{2} \phi^{2}+\int u u_{t} \phi^{2} \leq 2 \int|\nabla u||\nabla \phi||\phi||u|+\int|V| u^{2} \phi^{2}
$$

Since an integration by parts gives

$$
\int u u_{t} \phi^{2}=\frac{1}{2} \int\left(u^{2}\right)_{t} \phi^{2}=-\int u^{2} \phi \phi_{t},
$$

we obtain from (3.1)

$$
\int|\nabla u|^{2} \phi^{2} \leq 2 \int|\nabla u||\nabla \phi||\phi||u|+\int|V| u^{2} \phi^{2}+\int u^{2}|\phi|\left|\phi_{t}\right| .
$$

By the Cauchy-Schwarz inequality we have in a standard fashion $2 \int|\nabla u\|\nabla \phi\| \phi \| u| \leq$ $\frac{1}{2} \int|\nabla u|^{2} \phi^{2}+2 \int u^{2}|\nabla \phi|^{2}$. Substitution in the latter inequality gives

$$
\int|\nabla u|^{2} \phi^{2} \leq 4 \int u^{2}|\nabla \phi|^{2}+2 \int|V| u^{2} \phi^{2}+2 \int u^{2}|\phi|\left|\phi_{t}\right| .
$$

Using the bounds $|\nabla \phi| \leq C_{2} /|x|,\left|\phi_{t}\right| \leq C_{3} / T$, and the fact that $\phi, \nabla \phi, \phi_{t}$ are supported in $\{r(1-a) / 2<|x|<b r\} \times(-T, T)$, we obtain from (3.2) that the following holds,

$$
\int|\nabla u|^{2} \phi^{2} \leq \frac{C_{1}}{r^{2}} \int_{\{r(1-a) / 2<|x|<b r\} \times(-T, T)} u^{2},
$$

for some $C_{1}$ depending on $n, a, b, T$ and $M$. The desired conclusion follows by bounding from below the integral in the left-hand side with one over the region where $\phi \equiv 1$. 
Proof of Theorem 1.2 Throughout the proof the letter $C$ will indicate an all purpose constant which might change from line to line, and which could depend in some occurrences on the number $T$. In what follows it will be easier for the computations if we work with the symmetric time-interval $(-T, T)$, instead of $(0, T)$. Without loss of generality, we also assume that $R<1$. Let $0<r_{1}<r_{2} / 2<4 r_{2}<r_{3}<R / 2$ be fixed, and let $\phi(x)$ be a smooth function such that $\phi(x) \equiv 0$ when $|x|<r_{1} / 2$, $\phi(x) \equiv 1$ when $r_{1}<|x|<r_{2}, \phi(x) \equiv 0$ when $|x|>r_{3}$. We now let $T_{2}=T / 2$ and $T_{1}=3 T / 4$, so that $0<T_{2}<T_{1}<T$. As in [9], we let $\eta(t)$ be a smooth even function such that $\eta(t) \equiv 1$ when $|t|<T_{2}, \eta(t) \equiv 0$, when $|t|>T_{1}$. Furthermore, it will be important in the sequel (see (3.15) below) that $\eta$ decay exponentially near $t= \pm T_{1}$. As in (118) of [9] we take

$$
\eta(t)=\left\{\begin{array}{l}
0 \quad-T \leq t \leq-T_{1} \\
\exp \left(-\frac{T^{3}\left(T_{2}+t\right)^{4}}{\left(T_{1}+t\right)^{3}\left(T_{1}-T_{2}\right)^{4}}\right) \quad-T_{1} \leq t \leq-T_{2}, \\
1, \quad t \in-T_{2} \leq t \leq 0 .
\end{array}\right.
$$

We suppose that $u$ parabolically vanishes to infinite order in the sense of (1.4), and we want to conclude that $u \equiv 0$ in $B_{R} \times(-T, T)$. We assume that this not the case and show that we reach a contradiction. Without loss of generality we can (and will) assume that

$$
\int_{B_{r_{2}} \times\left(-T_{2}, T_{2}\right)} u^{2} \neq 0
$$

Otherwise, the result in [9] implies $u \equiv 0$ in $B_{R} \times\left(-T_{2}, T_{2}\right)$ and by the arguments that follow we could conclude that $u \equiv 0$ also for $|t|>T_{2}$. The assumption (3.4) will be used in the very end in the Eq. (3.24).

Now, with $u$ as in Theorem 1.2 we let $v=\phi \eta u$. A standard limiting argument allows to use such $v$ in the Carleman estimate (1.3), obtaining

$$
\alpha \int r^{-2 \alpha-4} e^{2 \alpha r^{\varepsilon}} v^{2}+\alpha^{3} \int r^{-2 \alpha-4+\varepsilon} e^{2 \alpha r^{\varepsilon}} v^{2} \leq C \int r^{-2 \alpha} e^{2 \alpha r^{\varepsilon}}\left(\Delta v-v_{t}\right)^{2} .
$$

Here, we have fixed some $\varepsilon \in(0, \varepsilon(n))$, where $\varepsilon(n)$ is as in the hypothesis of Theorem 1.1. Keeping in mind that

$$
\Delta v-v_{t}=\phi \eta\left(\Delta u-u_{t}\right)+u\left(\eta \Delta \phi-\phi \eta_{t}\right)+2 \eta<\nabla u, \nabla \phi>,
$$

we obtain

$$
\begin{aligned}
& \alpha \int r^{-2 \alpha-4} e^{2 \alpha r^{\varepsilon}} v^{2}+\alpha^{3} \int r^{-2 \alpha-4+\varepsilon} e^{2 \alpha r^{\varepsilon}} v^{2} \leq C \int r^{-2 \alpha} e^{2 \alpha r^{\varepsilon}}\left(\Delta u-u_{t}\right)^{2} \phi^{2} \eta^{2} \\
& \quad+C \int r^{-2 \alpha} e^{2 \alpha r^{\varepsilon}}\left(|\nabla u|^{2}|\nabla \phi|^{2}+u^{2}(\Delta \phi)^{2}\right) \eta^{2}+u^{2} \phi^{2} \eta_{t}^{2}
\end{aligned}
$$


Since the differential inequality (1.1) gives

$$
C \int r^{-2 \alpha} e^{2 \alpha r^{\varepsilon}}\left(\Delta u-u_{t}\right)^{2} \phi^{2} \eta^{2} \leq C M^{2} \int r^{-2 \alpha-4} e^{2 \alpha r^{\varepsilon}} v^{2}
$$

if we choose

$$
\alpha \geq 2 C M^{2}
$$

then the first integral in the right-hand side of (3.5) can be absorbed in the left-hand side. Consequently, from the way $\phi$ and $\eta$ have been chosen, and bearing in mind that $\nabla \phi$ is supported in $\left\{r_{1} / 2<r<r_{1}\right\} \cup\left\{r_{2}<r<r_{3}\right\}$ and that we have in such a set $|\nabla \phi|=O(1 / r),|\Delta \phi|=O\left(1 / r^{2}\right)$, we obtain from (3.5)

$$
\begin{aligned}
& \alpha \int r^{-2 \alpha-4} e^{2 \alpha r^{\varepsilon}} v^{2} \leq C \int_{\left\{r_{1} / 2<r<r_{1}\right\} \times\left(-T_{1}, T_{1}\right)} e^{2 \alpha r^{\varepsilon}}\left(r^{-2 \alpha-4} u^{2}+r^{-2 \alpha-2}|\nabla u|^{2}\right) \\
& \quad+C r_{2}^{-2 \alpha-4} e^{2 \alpha r_{2}^{\varepsilon}} \int_{\left\{r_{2}<r<r_{3}\right\} \times\left(-T_{1}, T_{1}\right)}\left(u^{2}+|\nabla u|^{2}\right) \\
& \quad+C \int_{\left\{r_{1} / 2<|x|<r_{3}\right\} \times\left(-T_{1}, T_{1}\right)} r^{-2 \alpha} e^{2 \alpha r^{\varepsilon}} u^{2} \phi^{2} \eta_{t}^{2}-2 \alpha^{3} \int r^{-2 \alpha-4+\varepsilon} e^{2 \alpha r^{\varepsilon}} v^{2}
\end{aligned}
$$

In (3.7), we have also used the fact that, since the functions

$$
r \rightarrow r^{-2 \alpha-4} e^{2 \alpha r^{\varepsilon}} \quad \text { and } \quad r \rightarrow r^{-2 \alpha} e^{2 \alpha r^{\varepsilon}}
$$

are decreasing in $(0,1)$, we can estimate

$$
\begin{aligned}
& \int_{\left\{r_{2}<r<r_{3}\right\} \times\left(-T_{1}, T_{1}\right)} r^{-2 \alpha} e^{2 \alpha r^{\varepsilon}}\left(u^{2}(\Delta \phi)^{2}+|\nabla u|^{2}|\nabla \phi|^{2}\right) \\
& \quad \leq C r_{2}^{-2 \alpha-4} e^{2 \alpha r_{2}^{\varepsilon}} \int_{\left\{r_{2}<r<r_{3}\right\} \times\left(-T_{1}, T_{1}\right)}\left(u^{2}+|\nabla u|^{2}\right) .
\end{aligned}
$$

We now split the second to the last term in the right-hand side of (3.7) in three parts

$$
\begin{aligned}
& C \int_{\left\{r_{1} / 2<|x|<r_{3}\right\} \times\left(-T_{1}, T_{1}\right)} r^{-2 \alpha} e^{2 \alpha r^{\varepsilon}} u^{2} \phi^{2} \eta_{t}^{2}=C \int_{\left\{r_{1} / 2<|x|<r_{1}\right\} \times\left(-T_{1}, T_{1}\right)} r^{-2 \alpha} e^{2 \alpha r^{\varepsilon}} u^{2} \phi^{2} \eta_{t}^{2} \\
& +C \int_{\left\{r_{1}<|x|<r_{2}\right\} \times\left(-T_{1}, T_{1}\right)} r^{-2 \alpha} e^{2 \alpha r^{\varepsilon}} u^{2} \phi^{2} \eta_{t}^{2}+C \int_{\left\{r_{2}<|x|<r_{3}\right\} \times\left(-T_{1}, T_{1}\right)} r^{-2 \alpha} e^{2 \alpha r^{\varepsilon}} u^{2} \phi^{2} \eta_{t}^{2}
\end{aligned}
$$

Since $\left|\eta_{t}\right| \leq C / T$, the first and third terms in the right-hand side of (3.9) are, respectively, estimated as follows using (3.8) 


$$
\begin{aligned}
& C \int_{\left\{r_{1} / 2<|x|<r_{1}\right\} \times\left(-T_{1}, T_{1}\right)} r^{-2 \alpha} e^{2 \alpha r^{\varepsilon}} u^{2} \phi^{2} \eta_{t}^{2} \leq C\left(\frac{r_{1}}{2}\right)^{-2 \alpha} e^{\frac{2 \alpha r_{1}}{2}} \int_{\left\{r_{1} / 2<|x|<r_{1}\right\} \times\left(-T_{1}, T_{1}\right)} u^{2} \\
& \leq C\left(\frac{r_{1}}{2 C_{1}}\right)^{-2 \alpha} \int_{\left\{r_{1} / 2<|x|<r_{1}\right\} \times\left(-T_{1}, T_{1}\right)} u^{2}
\end{aligned}
$$

where in the last inequality in (3.10), we have used the fact that $e^{\frac{2 \alpha r_{1}}{2}} \leq e^{2 \alpha r_{3}} \leq C_{1}^{2 \alpha}$, for some $C_{1}>0$ depending only on $r_{3}$, which has been fixed. Similarly, we have

$$
C \int_{\left\{r_{2}<|x|<r_{3}\right\} \times\left(-T_{1}, T_{1}\right)} r^{-2 \alpha} e^{2 \alpha r^{\varepsilon}} u^{2} \phi^{2} \eta_{t}^{2} \leq C r_{2}^{-2 \alpha} e^{2 \alpha r_{2}^{\varepsilon}} \int_{\left\{r_{2}<|x|<r_{3}\right\} \times\left(-T_{1}, T_{1}\right)} u^{2}
$$

In order to estimate the second term in the right-hand side of (3.9), we combine it with the last integral in the right-hand side of (3.7) and observe that, since $\phi \equiv 1$ in the region $\left\{r_{1}<|x|<r_{2}\right\}$, and the function $\eta_{t}$ is supported in the set $\left(-T_{1},-T_{2}\right) \cup\left(T_{2}, T_{1}\right)$, if we indicate $U=\left\{r_{1}<|x|<r_{2}\right\} \times\left[\left(-T_{1},-T_{2}\right) \cup\left(T_{2}, T_{1}\right)\right]$, we can bound

$$
\begin{aligned}
& C \int_{\left\{r_{1}<|x|<r_{2}\right\} \times\left(-T_{1}, T_{1}\right)} r^{-2 \alpha} e^{2 \alpha r^{\varepsilon}} u^{2} \phi^{2} \eta_{t}^{2}-2 \alpha^{3} \int r^{-2 \alpha-4+\varepsilon} e^{2 \alpha r^{\varepsilon}} v^{2} \\
& \quad \leq \int_{U} r^{-2 \alpha-4+\varepsilon} e^{2 \alpha r^{\varepsilon}} u^{2} \eta^{2}\left(C r^{4-\varepsilon} \frac{\eta_{t}^{2}}{\eta^{2}}-2 \alpha^{3}\right) \\
& \quad \leq \int_{U} r^{-2 \alpha-4+\varepsilon} e^{2 \alpha r^{\varepsilon}} u^{2} \eta^{2}\left(C r^{3} \frac{\eta_{t}^{2}}{\eta^{2}}-2 \alpha^{3}\right)
\end{aligned}
$$

Note that in the last inequality above, we used that for $\varepsilon<1$, we have $r^{4-\varepsilon}<r^{3}$, for all $r<1$. At this point our objective is to establish the following estimate

$$
\int_{U} r^{-2 \alpha-4+\varepsilon} e^{2 \alpha r^{\varepsilon}} u^{2} \eta^{2}\left(C r^{3} \frac{\eta_{t}^{2}}{\eta^{2}}-2 \alpha^{3}\right) \leq C \int_{B_{R} \times(-T, T)} u^{2} .
$$

The proof of (3.12) will be accomplished in several steps. First, we note that it suffices to concern ourselves with the portion of the integral in the left-hand side of (3.12) over the region $U^{-}=\left\{r_{1}<|x|<r_{2}\right\} \times\left(-T_{1},-T_{2}\right)$, since the estimate on $U^{+}=$ $\left\{r_{1}<|x|<r_{2}\right\} \times\left(T_{2}, T_{1}\right)$ is similar. Now, if $-T_{1} \leq t \leq-T_{2}$, keeping in mind that $T_{1}-T_{2}=\frac{T}{4},\left|T_{2}+t\right| \leq T_{1}-T_{2}=\frac{T}{4}$, and that $\frac{3}{4} T \leq 4 T_{1}-3 T_{2}+t \leq T$, from (3.3) a standard calculation shows

$$
\left|\frac{\eta_{t}}{\eta}\right|=\left|\frac{T^{3}\left(T_{2}+t\right)^{3}\left(4 T_{1}-3 T_{2}+t\right)}{\left(T_{1}-T_{2}\right)^{4}\left(T_{1}+t\right)^{4}}\right| \leq \frac{4 T^{3}}{\left|T_{1}+t\right|^{4}} .
$$

Using this estimate in the above inequality, we obtain 


$$
\begin{aligned}
& \int_{U^{-}} e^{2 \alpha r^{\varepsilon}} r^{-2 \alpha-4+\varepsilon} u^{2} \eta^{2}\left(C r^{3} \frac{\eta_{t}^{2}}{\eta^{2}}-2 \alpha^{3}\right) \\
& \quad \leq \int_{U^{-}} e^{2 \alpha r^{\varepsilon}} r^{-2 \alpha-4+\varepsilon} u^{2} \eta^{2}\left(C r^{3} \frac{T^{6}}{\left(T_{1}+t\right)^{8}}-2 \alpha^{3}\right) .
\end{aligned}
$$

Next, we write $U^{-}=D \cup\left(U^{-} \backslash D\right)$, where $D$ is the region in $U^{-}$where the inequality

$$
2 \alpha^{3} \leq C r^{3} \frac{T^{6}}{\left(T_{1}+t\right)^{8}}
$$

holds. Since we clearly have $\int_{U^{-} \backslash D} e^{2 \alpha r^{\varepsilon}} r^{-2 \alpha-3} u^{2} \eta^{2}\left(C r^{3} \frac{T^{6}}{\left(T_{1}+t\right)^{8}}-2 \alpha^{3}\right) \leq 0$, we obtain

$$
\begin{aligned}
& \int_{U^{-}} e^{2 \alpha r^{\varepsilon}} r^{-2 \alpha-4+\varepsilon} u^{2} \eta^{2}\left(C r^{3} \frac{\eta_{t}^{2}}{\eta^{2}}-2 \alpha^{3}\right) \\
& \leq \int_{D} e^{2 \alpha r^{\varepsilon}} r^{-2 \alpha-4+\varepsilon} u^{2} \eta^{2}\left(C r^{3} \frac{T^{6}}{\left(T_{1}+t\right)^{8}}-2 \alpha^{3}\right) \\
& \leq C \int_{D} e^{2 \alpha r^{\varepsilon}} r^{-2 \alpha-4+\varepsilon} \eta u^{2} \frac{\eta T^{6}}{\left(T_{1}+t\right)^{8}} .
\end{aligned}
$$

Comparing the right-hand side of (3.14) with that of (3.12), it should be clear to the reader that, in order to establish (3.12), it suffices at this point to be able to bound from above in $D$ the quantity $r^{-2 \alpha-4+\varepsilon} e^{2 \alpha r^{\varepsilon}} \eta \frac{\eta T^{6}}{\left(T_{1}+t\right)^{8}}$. We accomplish this by first observing that, thanks to the exponential vanishing of $\eta$ at $t=-T_{1}$, see (3.3), we obtain for $t \in\left(-T_{1},-T_{2}\right)$,

$$
\frac{\eta T^{6}}{\left(T_{1}+t\right)^{8}} \leq C,
$$

for some universal $C>0$ (depending on $T$ ). Secondly, we show that, thanks to the inequality (3.13), the following holds in the region $D$ provided that we choose the parameter $\alpha$ large enough

$$
r^{-2 \alpha-4+\varepsilon} e^{2 \alpha r^{\varepsilon}} \eta \leq 1 \text {. }
$$

Using the expression (3.3) for $\eta(t)$, we see that (3.16) does hold in $D$ if and only if for $\alpha$ sufficiently large we have in such set

$$
(2 \alpha+4-\varepsilon) \log r+\frac{T^{3}\left(T_{2}+t\right)^{4}}{\left(T_{1}+t\right)^{3}\left(T_{1}-T_{2}\right)^{4}}-2 \alpha r^{\varepsilon} \geq 0 .
$$

To prove (3.17) observe that (3.13) can be equivalently written in $D$ as

$$
\frac{T_{1}+t}{T} \leq\left(\frac{C}{2 T^{2}}\right)^{1 / 8}\left(\frac{r}{\alpha}\right)^{3 / 8}=C\left(\frac{r}{\alpha}\right)^{3 / 8},
$$


for some universal $C>0$. Since for $\alpha$ sufficiently large we trivially have

$$
C\left(\frac{r}{\alpha}\right)^{3 / 8} \leq C\left(\frac{R}{\alpha}\right)^{3 / 8} \leq \frac{1}{12}
$$

we conclude that in $D$ we must have

$$
\frac{T_{1}+t}{T} \leq \frac{1}{12}
$$

if $\alpha>1$ has been chosen large enough. Since $\frac{T}{4}=T_{1}-T_{2}=T_{1}+t+\left|T_{2}+t\right|$, from (3.18) we conclude that we must have in $D$

$$
\left|T_{2}+t\right| \geq \frac{T}{6}
$$

If we now use this bound from below along with (3.13), we find in $D$

$$
\begin{aligned}
& (2 \alpha+4-\varepsilon) \log r+\frac{T^{3}\left(T_{2}+t\right)^{4}}{\left(T_{1}+t\right)^{3}\left(T_{1}-T_{2}\right)^{4}}-2 \alpha r^{\varepsilon} \\
& \geq\left(\frac{4}{6}\right)^{4}\left(\frac{2}{C}\right)^{3 / 8} T^{3 / 4}\left(\frac{\alpha}{r}\right)^{9 / 8}-(2 \alpha+4-\varepsilon) \log \frac{1}{r} \\
& \quad-2 \alpha r^{\varepsilon}=C\left(\frac{\alpha}{r}\right)^{9 / 8}-(2 \alpha+4-\varepsilon) \log \frac{1}{r}-2 \alpha r^{\varepsilon} \geq 0,
\end{aligned}
$$

provided that $r<r_{3} \leq 1$, and that $\alpha$ is sufficiently large. We stress here the critical role of the power $\alpha^{9 / 8}$, versus the linear term $2 \alpha+4-\varepsilon$, in reaching the above conclusion. This is precisely why we needed to incorporate the subcritical term $\alpha^{3} \int r^{-2 \alpha-4+\varepsilon} e^{2 \alpha r^{\varepsilon}} u^{2}$ in our main Carleman estimate (1.3). We have thus proved (3.17), and consequently (3.16). Combining (3.14), (3.15) and (3.16), we conclude that (3.12) holds.

Using now the estimates (3.9), (3.10), (3.11) and (3.12) in (3.7), we find

$$
\begin{aligned}
& \alpha \int r^{-2 \alpha-4} e^{2 \alpha r^{\varepsilon}} v^{2} \leq C \int_{\left\{r_{1} / 2<|x|<r_{1}\right\} \times\left(-T_{1}, T_{1}\right)} e^{2 \alpha r^{\varepsilon}}\left(r^{-2 \alpha-4} u^{2}+r^{-2 \alpha-2}|\nabla u|^{2}\right) \\
& +C r_{2}^{-2 \alpha-4} e^{2 \alpha r_{2}^{\varepsilon}} \int_{\left\{r_{2}<|x|<r_{3}\right\} \times\left(-T_{1}, T_{1}\right)}\left(u^{2}+|\nabla u|^{2}\right) \\
& +C\left(\frac{r_{1}}{C_{2}}\right)^{-2 \alpha} \int_{\left\{r_{1} / 2<|x|<r_{1}\right\} \times\left(-T_{1}, T_{1}\right)} u^{2}+C \int_{B_{R} \times(-T, T)} u^{2} .
\end{aligned}
$$

Note that in (3.20) we have let $C_{2}=2 C_{1}$, with $C_{1}$ as in (3.10).

Now by Lemma 3.1 and (3.8) it follows that for some universal $C_{4}$,

$$
\int_{\left\{r_{1} / 2<|x|<r_{1}\right\} \times\left(-T_{1}, T_{1}\right)} r^{-2 \alpha-2} e^{2 \alpha r^{\varepsilon}}|\nabla u|^{2} \leq C\left(\frac{r_{1}}{C_{4}}\right)^{-2 \alpha-4} \int_{\left\{r_{1} / 4<|x|<3 r_{1} / 2\right\} \times(-T, T)} u^{2},
$$


and also

$$
\int_{\left\{r_{2}<|x|<r_{3}\right\} \times\left(-T_{1}, T_{1}\right)}|\nabla u|^{2} \leq C \int_{B_{R} \times(-T, T)} u^{2},
$$

where the constant in the latter estimate depends also on the parameters $r_{2}<r_{3} \leq 1$, which are finally fixed at this point. Substituting these bounds in (3.20), we conclude that the following inequality holds for some new universal constants $C$ and $C_{1}$,

$$
\begin{aligned}
& \alpha \int r^{-2 \alpha-4} e^{2 \alpha r^{\varepsilon}} v^{2} \leq C\left(\frac{r_{1}}{C_{1}}\right)^{-2 \alpha-4} \int_{\left\{r_{1} / 4<|x|<3 r_{1} / 2\right\} \times(-T, T)} u^{2} \\
& +C r_{2}^{-2 \alpha-4} e^{2 \alpha r_{2}^{\varepsilon}} \int_{B_{R} \times(-T, T)} u^{2} .
\end{aligned}
$$

The integral in the left-hand side of (3.21) can be bounded from below in the following way using (3.8),

$$
\alpha \int r^{-2 \alpha-4} e^{2 \alpha r^{\varepsilon}} v^{2} \geq \alpha r_{2}^{-2 \alpha-4} e^{2 \alpha r_{2}^{\varepsilon}} \int_{\left\{r_{1}<|x|<r_{2}\right\} \times\left(-T_{2}, T_{2}\right)} u^{2} .
$$

Substituting (3.22) in (3.21), and dividing both sides by $r_{2}^{-2 \alpha-4} e^{2 \alpha r_{2}^{\varepsilon}}$, we obtain

$$
\alpha \int_{\left\{r_{1}<|x|<r_{2}\right\} \times\left(-T_{2}, T_{2}\right)} u^{2} \leq C\left(\frac{r_{1}}{C_{1} r_{2}}\right)^{-2 \alpha-4} \int_{\left\{r_{1} / 4<|x|<3 r_{1} / 2\right\} \times(-T, T)} u^{2}+C \int_{B_{R} \times(-T, T)} u^{2} .
$$

Now adding $\alpha \int_{B_{r_{1}} \times\left(-T_{2}, T_{2}\right)} u^{2}$ to both sides of the latter inequality, we obtain

$$
\begin{aligned}
& \alpha \int_{B_{r_{2}} \times\left(-T_{2}, T_{2}\right)} u^{2} \leq C\left(\frac{r_{1}}{C_{1} r_{2}}\right)^{-2 \alpha-4} \int_{\left\{r_{1} / 4<|x|<3 r_{1} / 2\right\} \times(-T, T)} u^{2} \\
& +\alpha \int_{B_{r_{1}} \times\left(-T_{2}, T_{2}\right)} u^{2}+C \int_{B_{R} \times(-T, T)} u^{2} \\
& \quad \leq 2 C\left(\frac{r_{1}}{C_{1} r_{2}}\right)^{-2 \alpha-4} \int_{B_{3 r_{1} / 2 \times(-T, T)}} u^{2}+C \int_{B_{R} \times(-T, T)} u^{2},
\end{aligned}
$$

where, recalling our initial choice $r_{1}<r_{2}$, we note that in the second inequality in (3.23) we have used

$$
\alpha \leq\left(\frac{r_{1}}{C_{2} r_{2}}\right)^{-2 \alpha-4}
$$

Keeping in mind the hypothesis (3.4), we now choose $\alpha$ (depending on $u$ ) such that

$$
\alpha \int_{B_{r_{2}} \times\left(-T_{2}, T_{2}\right)} u^{2} \geq 2 C \int_{B_{R} \times(-T, T)} u^{2}
$$


where $C$ is as in (3.23). Thus, by subtracting off $C \int_{B_{R} \times(-T, T)} u^{2}$ from both sides of (3.23), we obtain

$$
\left(\frac{r_{1}}{C_{1} r_{2}}\right)^{2 \alpha+4} \frac{\alpha}{2} \int_{B_{r_{2}} \times\left(-T_{2}, T_{2}\right)} u^{2} \leq 2 C \int_{B_{3 r_{1} / 2} \times(-T, T)} u^{2} .
$$

At this point, we fix $\alpha$ sufficiently large in such a way that (3.6), (3.18), (3.19) and (3.24) simultaneously hold. Letting $3 r_{1} / 2=s$, we obtain from (3.25) that for some new constants $C, A$ depending on $r_{2}, r_{3}, R$, the ratio $\frac{\int_{B_{R} \times(-T, T)} u^{2}}{\int_{B_{r_{2}} \times\left(-T_{2}, T_{2}\right)} u^{2}}$, and $\alpha$ (which at this point is fixed), the following holds for all $0<s \leq r_{2} / 8$,

$$
\int_{B_{s} \times(-T, T)} u^{2} \geq C s^{A} .
$$

Since this estimate is in contradiction with the hypothesis that $u$ parabolically vanish to infinite order in the sense of (1.4), we have finally proved the theorem.

Acknowledgements Open access funding provided by Università degli Studi di Padova within the CRUICARE Agreement. This work began during a visit of the second named author to the Tata Institute of Fundamental Research, Centre For Applicable Mathematics, in Bangalore, during February 2020. He gratefully acknowledges the gracious hospitality of the Centre and the warm work environment.

Open Access This article is licensed under a Creative Commons Attribution 4.0 International License, which permits use, sharing, adaptation, distribution and reproduction in any medium or format, as long as you give appropriate credit to the original author(s) and the source, provide a link to the Creative Commons licence, and indicate if changes were made. The images or other third party material in this article are included in the article's Creative Commons licence, unless indicated otherwise in a credit line to the material. If material is not included in the article's Creative Commons licence and your intended use is not permitted by statutory regulation or exceeds the permitted use, you will need to obtain permission directly from the copyright holder. To view a copy of this licence, visit http://creativecommons.org/licenses/by/4.0/.

\section{References}

1. Baras, P., Goldstein, J.A.: The heat equation with a singular potential. Trans. Am. Math. Soc. 284(1), 121-139 (1984)

2. Escauriaza, L., Vessella, S.: Optimal three cylinder inequalities for solutions to parabolic equations with Lipschitz leading coefficients. Contemp. Math. 333, 79-87 (2003)

3. Garofalo, N., Lin, F.: Monotonicity properties of variational integrals, $A_{p}$-weights and unique continuation. Indiana Univ. Math. J. 35, 245-268 (1986)

4. Jerison, D., Kenig, C.: Unique continuation and absence of positive eigenvalues for Schrödinger operators. With an appendix by E. M. Stein. Ann. Math. 121(3), 463-494 (1985)

5. Hörmander, L.: Uniqueness theorems for second order elliptic differential equations. Commun. Partial Differ. Equ. 8(1), 21-64 (1983)

6. Lin, C., Nakamura, G., Wang, J.: Quantitative uniqueness for second order elliptic operators with strongly singular coefficients. Rev. Mat. Iberoam. 27, 475-491 (2011)

7. Pan, Y.: Unique continuation for Schrodinger operators with singular potentials. Commun. Partial Differ. Equ. 17(5-6), 953-965 (1992)

8. Regbaoui, R.: Strong uniqueness for second order differential operators. J. Differ. Equ. 141(2), 201-217 (1997) 
9. Vessella, S.: Carleman estimates, optimal three cylinder inequality, and unique continuation properties for solutions to parabolic equations. Commun. Partial Differ. Equ. 28(3-4), 637-676 (2003)

Publisher's Note Springer Nature remains neutral with regard to jurisdictional claims in published maps and institutional affiliations. 